\title{
Evaluación De Métodos Indirectos Para Estimar Biomasa En Un Pastizal Natural Del Sur De Córdoba (Argentina Central)
}

\author{
Mónaco N. \\ V. Santa \\ M.J. Rosa
}

Departamento Ecología Agraria. Facultad de Agronomía y Veterinaria

V. Autran

Departamento Biología Agrícola, UNRC, Córdoba, Argentina

Doi: 10.19044/esj.2017.v13n36p59 URL:http://dx.doi.org/10.19044/esj.2017.v13n36p59

\begin{abstract}
To optimize grassland management, it is important to evaluate seasonal changes in quality and production according to the characteristics of the environment, allowing planning and rational use of resources. With the objective of finding estimators that fit the actual production of aerial biomass in natural pastures, it was proposed to estimate biomass with the average height of the pasture and by double sampling, by determination by direct weight estimation and by estimation with index assignment. For this purpose, a natural grassland located in the area of influence of the final section of the Chucul River Basin (La Felipa Natural Reserve) was surveyed. Periodically from April 2013 to March 2016, in ten samples taken with 0.25 $\mathrm{m}^{2}$ quadrants, the biomass was cut and weighed, and the height of the present species was measured. In the area surrounding each quadrant, 50 uncorrected visual observations were made by assigning indexes from 1 to 5 and values of estimated weight according to the amount of forage available. Then a correlation analysis was performed and linear regressions were determined between the parameters studied. Real biomass (BR) showed a significant correlation with biomass estimated by Weight (BEP) and biomass estimated by Index (BEI) $(\mathrm{p} \leq 0.05)$.
\end{abstract}

Keywords: Natural grassland, estimation by index, estimation by weight, estimation by height. 


\section{Resumen}

Para optimizar el manejo de los pastizales es relevante evaluar cambios estacionales en la calidad y la producción en función de las características del medio ambiente, que permitan la planificación y el uso racional de los recursos. Con el objetivo de encontrar estimadores que se ajusten a la producción real de biomasa aérea en pastizales naturales se propuso estimar biomasa con la altura promedio del pastizal y por doble muestreo, mediante determinación por estimación directa de peso y por estimación con asignación de indices. Para ello se realizó el relevamiento de un pastizal natural ubicado en el área de influencia del trayecto final de la cuenca del arroyo Chucul (Reserva Natural La Felipa). Periódicamente de abril de 2013 a marzo de 2016, en diez muestras tomadas con cuadrantes de $0,25 \mathrm{~m}^{2}$, se cortó y se pesó la biomasa, y se midió la altura de las especies presentes. En el área circundante a cada cuadrante, se realizaron 50 observaciones visuales sin cortar asignándole índices del 1 al 5 y valores de peso estimado según la cantidad de forraje disponible. Luego se realizó un análisis de correlación y se determinaron regresiones lineales entre los parámetros estudiados. La biomasa real (BR) mostró una correlación significativa con la biomasa estimada por peso (BEP) y la biomasa estimada por índice $(\mathrm{BEI})(\mathrm{p} \leq 0.05)$.

Palabras-claves: Pastizal natural, estimación por índice, estimación por peso, estimación por altura.

\section{Introducción}

La superficie ocupada por pastizales naturales en Argentina corresponde a una amplia variedad de climas, suelos y vegetación; ocupando aproximadamente el $70 \%$ del territorio nacional (Chiossone, 2011). Los mismos están constituidos por comunidades de vegetación herbácea, montes y bosque nativos (INTA, 2011). Como recurso forrajero el pastizal es utilizado esencialmente para la cría de animales domésticos, brindando además servicios tales como hábitat de fauna, estabilidad de cuencas, provisión de agua y oxígeno y mantenimiento de la diversidad específica. El mal uso de los mismos puede afectar seriamente todas las funciones de estos ecosistemas (INTA, 2011).

A fin de optimizar el manejo de los pastizales es relevante evaluar cambios estacionales, calidad y la producción en función de las características del ambiente para la planificación y el uso racional de los recursos (Pueyo et al., 2005). Esta determinación puede realizarse con métodos directos (tradicional o destructiva) o indirectos. Los primeros consisten en cortar, secar y pesar el material (Fernández, 2004). Los 
segundos se basan en estimaciones visuales, medidas de altura, volúmenes de los pastos, fotografías, uso de imágenes satelitales (Medina et al., 2009; Moliterno, 1997). Diversos estudios científicos han utilizado diferentes métodos indirectos para estimar la biomasa y la productividad de relictos naturales (Brown, 1954; Singh et al., 1975; Redmann, 1992, Fernández, 2004; Millapán et al., 2009).

La elección de la metodología con la que se realizará el muestreo está condicionada por los objetivos que se persigan, las condiciones de medición, la disponibilidad de recursos humanos y materiales, la posibilidad de acceso a los lotes y las características de la vegetación, entre otros, (Valencia Gutiérrez, 2013).

La metodología tradicional (cosecha directa por corte) es en general precisa a nivel local (Sala et al., 1988; Sala y Austin, 2000; Scarabotti et al., 2002), pero insume tiempo y recursos que en muchas oportunidades son restrictivos (Gardner, 1967; Sala et al., 1988; Sala y Austin, 2000; Turner et al., 2001, Houghton et al., 2001) y presentan la desventaja de tener limitaciones en la extrapolación de los resultados a grandes áreas (Lillesand et al., 2014; Aronoff, 2005; Richards y Jia, 2006). De acuerdo a lo mencionado precedentemente y por provocar escaso o ningún daño en la vegetación, los métodos no destructivos, han cobrado gran importancia (t’Mannetje, 1978, 2000; Hidalgo et al., 1990; Sala y Austin, 2000; Díaz, 2007). La estimación de valores de biomasa se realiza utilizando regresiones y ecuaciones de ajustes a partir de variables cuantitativas (Ramírez Náder et al., 2008). En comunidades de pastizales se toman en cuenta variables cuantitativas de medidas como altura de las plantas, forma, cobertura y superficie de suelo desnudo (t’Mannetje, 1978, 2000; Millapán et al., 2009) para estimar la biomasa total o en particular de algunas especies (Reese et al., 1980; Andariese y Covington, 1986; Johnson et al., 1988; Guevara et al., 2002). Con el objetivo de encontrar estimadores que se ajusten a la producción real de biomasa aérea en pastizales naturales se propuso un proyecto de trabajo en el área de influencia del trayecto final de la cuenca del arroyo Chucul (Reserva Natural La Felipa) sobre estimación de biomasa a partir con de la altura promedio del pastizal y por doble muestreo. Este último mediante: a) determinación de biomasa por estimación directa de peso y b) estimación por observación con asignación de índices.

\section{Objetivos}

- Contrastar la biomasa medida por el método tradicional de corte y pesado, con la calculada a partir de la estimación por peso.

- Cotejar la biomasa medida por el método tradicional de corte y pesado, con la estimada a partir de índices. 
-Comparar la biomasa medida por el método tradicional de corte y pesado, con la estimada a partir de altura.

- Evaluar el nivel de ajuste existente entre la biomasa estimada por diferentes métodos indirectos y la biomasa real obtenida por el método tradicional.

\section{Materiales y Métodos}

\section{Ubicación geográfica:}

Este trabajo se desarrolló durante 3 años, desde abril de 2013 a marzo de 2016, en un campo ubicado a 15 kilómetros al sur de la localidad de Ucacha $\left(33^{\circ} 00^{\prime}-33^{\circ} 20^{\prime} \mathrm{S}\right.$ y $\left.6^{\circ} 30^{\prime}-64^{\circ} 00^{\prime} \mathrm{W}\right)$, departamento de Juárez Celman, provincia de Córdoba, República Argentina (Figura 1).

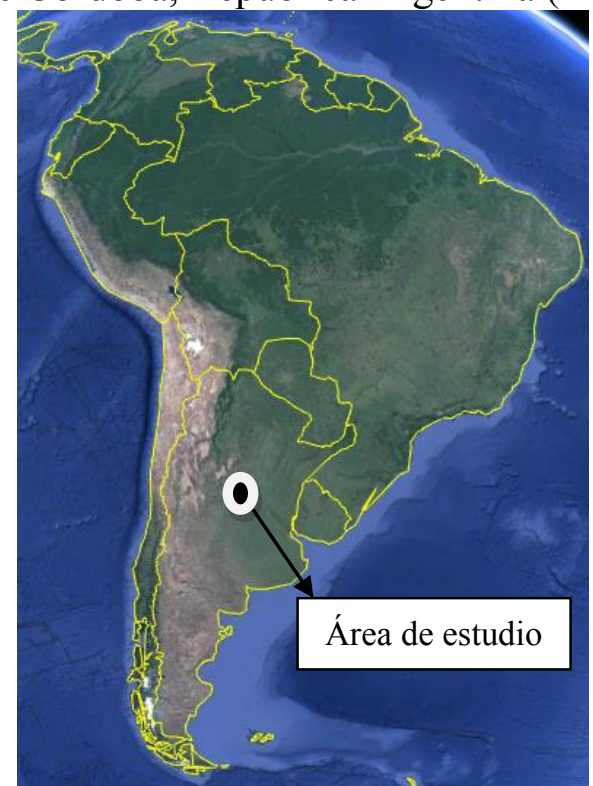

Figura 1: Área de estudio, Ucacha, Provincia de Córdoba (República Argentina). Fuente: Google Earth.

El estudios se llevó a cabo en un área de pastizales naturales, que es representativo por su extensión en la zona y por su interés agronómico respecto a las características de las especies que los conforman (Menghi et al; 2000).

El paisaje es de llanura pampeana arenosa y ondulada (Jarsun, 1993) caracterizado por zonas altas planas con suelos bien drenados aptos para uso agrícola generalizado y aproximadamente 12.000 has de zonas deprimidas con lagunas o anegamientos temporarios, de las cuales 7000 has tienen complejos de suelos hidrohalomórficos, drenaje impedido y la capa freática fluctuando próxima a la superficie (Jarsun, 1993). 
El clima es templado - subhúmedo, con una distribución de precipitaciones de tipo monzónico. En el transcurso del ensayo la precipitación media fue de $772 \mathrm{~mm}$, los valores medios de temperaturas anuales son de $16 \mathrm{C}^{\circ}$ en enero y $9,5 \mathrm{C}^{\circ}$ en junio (Menghi et al., 1998). El período libre de heladas medio es de 240 días (Rodríguez, 1997).

Desde el punto de vista florístico distintos autores (Cabrera 1976, León y Anderson 1983; y Burkart et al., 2000) coinciden en incluir a éste área como parte del "Distrito Pampeano Occidental de la Provincia Fitogeográfica Pampeana". A su vez, Luti et al., (1979) la considera dentro de la vegetación de Bañados y Lagunas, y Bianco et al. (1987) en el de llanuras bien drenadas. El área es un pastizal de gramíneas cespitosas perennes cuyos géneros dominantes son: Stipa, Piptochaetium, Poa, Briza, Setaria y Aristida, con presencia aislada de componentes leñosos. En las zonas deprimidas persiste la vegetación espontánea en estado seminatural con predominio de hidrófilas emergentes (géneros: Typha, Scirpus, Juncus y Eleocharis) donde la inundación es permanente y de comunidades halófilas (géneros: Distichlis, Salicornia, Poa y Muelembergia) en sitios en que se producen inundaciones temporales.

\section{Muestreo}

El muestreo a campo consistió en el relevamiento de información sobre altura, cobertura, biomasa aérea de la vegetación y proporción de suelo desnudo. Para aplicar el método de doble muestreo (Haydock y Shawn, 1975) y de estimación de biomasa por altura, se utilizó la asociación de observaciones visuales con datos obtenidos por muestreo directo a campo para determinar estimadores de regresión. Para definir condiciones dentro de la totalidad del sitio previamente se recorrió el área completa a estudiar. Se definieron zonas con volúmenes máximos, mínimos e intermedios de forrajimasa. En cada fecha se fijaron 10 unidades muestrales o cuadrados de referencia o corte de 0,50 x 0,50 m (Fernández, 2004). La cantidad de cuadrados de observación de estimaciones visuales y de altura se definió en 50 (Díaz 2007, Fernández 2004 y Cangiano 1996). En las áreas circundantes a cada una de las diez unidades de corte se registró la altura por especie en cinco unidades muestrales de, con regla desde la base de la planta e incluyendo la estructura reproductiva y a su vez, se realizaron al azar cinco observaciones visuales siguiendo dos patrones: a) indicando directamente una cifra aproximada de materia fresca en $\mathrm{g} / 0,25 \mathrm{~m}^{2}$ (biomasa estimada por peso: BEP), usando como criterios cobertura vegetal, porcentaje de suelo desnudo, altura y volumen de forraje para asignar valores máximos y mínimos (Gardner, 1967 y Carrillo y Fernández, 1988); b) utilizando categorías de índices del 1 al 5, donde 1 representa la mínima cantidad de biomasa que se espera encontrar y 5 la máxima, a la que se denominó 
biomasa estimada por índice: BEI (Díaz, 2007; Franco Quintero et al., 2006). Para los valores intermedios se calculó el promedio ponderado.

Periódicamente se cortó biomasa aérea en pie en diez muestras de $0,25 \mathrm{~m}^{2}$ ubicados al azar. El material cosechado fue colocado en estufa a $60^{\circ} \mathrm{C}$ hasta peso constante y posteriormente pesado para determinar peso seco real (BR).

Para predecir la biomasa estimada por altura: BEA, se registró altura promedio de las especies presentes y por medio de modelos de regresión lineal se estimó BEA (Millapán, 2006; Puchetta et al., 2004 y Fernández, 2004).

Con los valores estimados visualmente a campo y los valores de peso seco, utilizando la fórmula de Gardner (1967), se estimó BEP y BEI:

Dónde:

$$
\mathrm{X}=\mathrm{X}^{*}+\mathrm{b}^{*}\left(\mathrm{Y}-\mathrm{Y}^{*}\right)
$$

$\mathrm{X}^{\prime \prime}$ : media aritmética de los pesos secos

b: coeficiente de regresión

Y: media aritmética total de estimaciones visuales

$\mathrm{Y}^{*}$ : media aritmética de las estimaciones visuales correspondientes a las muestras cortadas y pesadas.

Se realizaron regresiones lineales entre datos apareados para cada uno de los métodos indirectos (estimación visual y regla graduada) y el método directo (peso seco de la biomasa total). Se generaron ecuaciones de calibración para cada una de las fechas de muestreo. El ajuste de los métodos indirectos con el método directo se evaluó a través de los coeficientes $\mathrm{R}^{2}$ de dichas regresiones (Millapán, 2006).

\section{Procesamiento de datos}

Los análisis de correlación de BR, BEI, BEP y BEA se realizaron utilizando el paquete estadístico Infostat- (Di Rienzo et al., 2010). Posteriormente se determinaron regresiones lineales de BEP vs. BR; BEI vs. BR y BEA vs BR.

\section{Resultados y Discusión}

En la en la Figura 2 se muestran los valores obtenidos de BR y los valores estimados por BEI, BEP y BEA. 


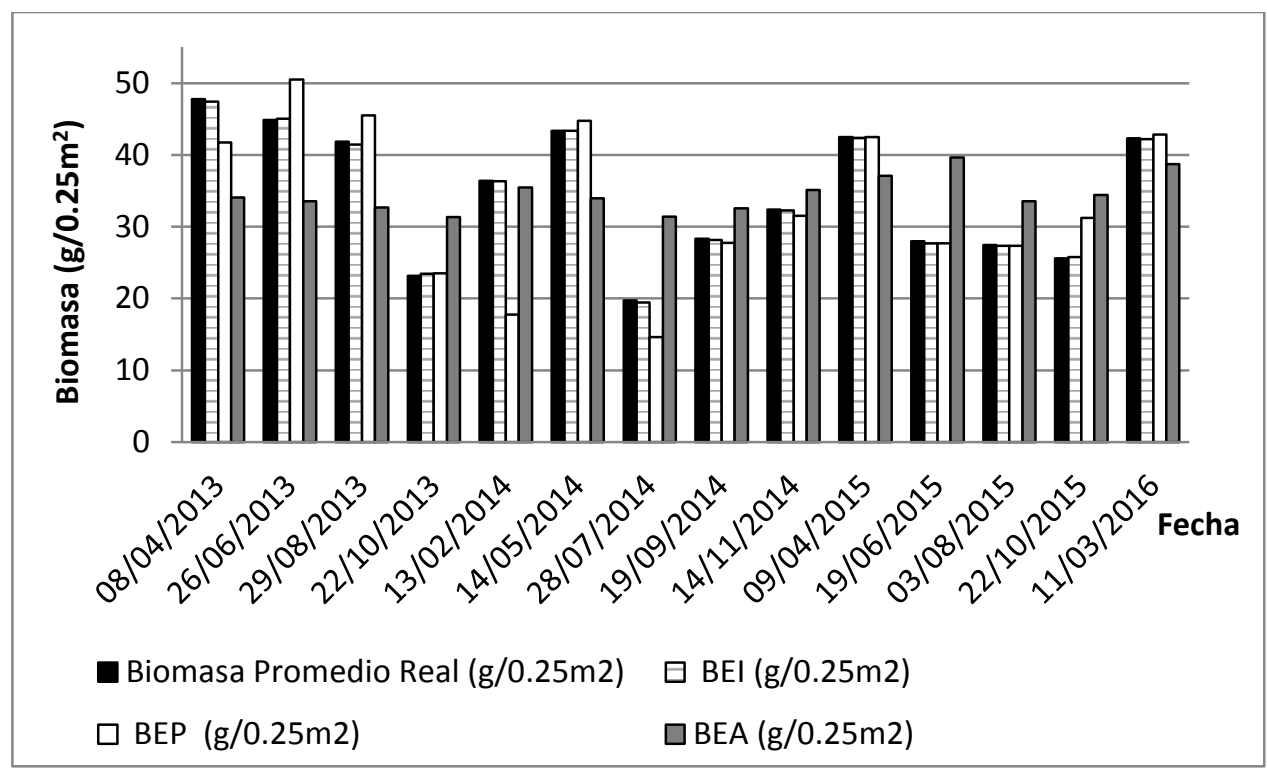

Figura 2: Valores de biomasa real, biomasa estimada por Índice (BEI), por Peso (BEP) y biomasa estimada por Altura (BEA) para el ciclo 2013-2016 para un pastizal natural en el sur de Cordoba, Argentina.

Como se puede observar, los valores oscilaron entre $23-48 \mathrm{~g} / 0,25 \mathrm{~m}^{2}$ para BR; $26-47 \mathrm{~g} / 0,25 \mathrm{~m}^{2}$ para BEI; $14-50 \mathrm{~g} / 0,25 \mathrm{~m}^{2}$ para BEP y $33-40$ $\mathrm{g} / 0,25 \mathrm{~m}^{2}$ para BEA. Durante todo el ciclo de estudio, los valores de BEI fueron los que más se aproximaron a la BR y los que menor oscilación presentaron. Las diferencias entre la BR y los índices calculados fluctuaron entre +0.33 y $-0,29$ para BEI, entre +18.67 y -5.66 para BEP y entre +13.71 y -11.62 para BA. Trabajos anteriores con iguales condiciones de crecimiento del pastizal tuvieron valores similares de BEI y BEP (Mónaco et al., 2014; 2015).

A partir del análisis de correlación de los parámetros estudiados (BR, BEI, BEP y BEA) se observa que BR se correlacionó significativamente con BEP y BEI (Tabla 1) y por lo tanto se podrían utilizar ambos métodos en áreas de difícil acceso y evitar disturbios en relictos naturales. En la determinación de índices y estimación de peso fresco a campo, se usan como parámetros biológicos cobertura vegetal, porcentaje de suelo desnudo, altura y volumen de forraje para asignar valores máximos y mínimos, y en la determinación por altura solo ese dato.

La utilización de la altura como único parámetro estimador limitaría la aplicación de este método de estimación de biomasa para pastizales con las condiciones y características de la zona en estudio coincidiendo con Millapán (2006). 
Tabla 1: Resultados del análisis de correlación de Pearson

\begin{tabular}{|c|c|c|c|}
\hline & BR & BEI & BEP \\
\hline BEI & $1.0 *^{*}$ & & \\
\hline BEP & $0.84^{*}$ & $0.84^{*}$ & \\
\hline BEA & 0.29 & 0.29 & 0.21 \\
\hline
\end{tabular}

$* \mathrm{p} \leq 0.05$

Del análisis de correlación de los parámetros estudiados (BR, BEI, BEP y BEA) se observa que BR se correlacionó significativamente con BEP y BEI, por lo tanto se podrían utilizar ambos métodos en áreas de difícil acceso y evitar disturbios en relictos naturales.

En la Figura 3 se observan que las regresiones entre BR vs. BEI y BR vs. BEP muestran valores aceptables de $\mathrm{R}^{2}$. El mayor nivel de ajuste entre BR vs. BEI se explicaría porque el método considera un mayor número de parámetros para su determinación, coincidiendo con Hidalgo et al., 1990 y Mónaco et al., 2015, quienes trabajaron con estimaciones realizadas por índice. Por otro lado el R2 de BR vs. BEA muestra valores no significativos, respecto a esto Millapán (2006) menciona que la desventaja del uso de la regla graduada es no contemplar cambios de la variación de la densidad del follaje ya que solamente registra altura.

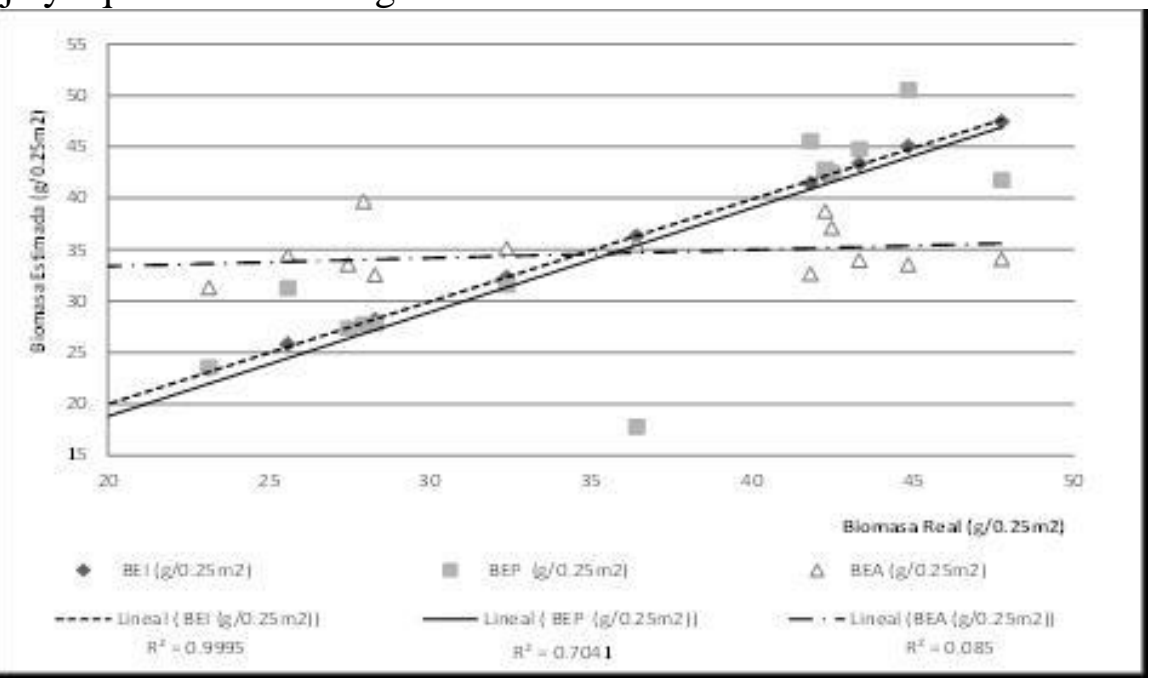

Figura 3: Regresión lineal simple entre BR vs. BEP, BR vs. BEI y BR vs. BEA para un pastizal natural en el sur de Córdoba, Argentina

\section{Conclusión}

La aplicación de estimadores como métodos indirectos para determinar biomasa aérea en pie en sistemas de pastizales naturales constituye una importante herramienta que disminuye los niveles de disturbios en áreas sensibles e incrementa la capacidad operativa de los 
equipos de investigación. Teniendo en cuenta un pastizal natural herbáceo con características cualitativas y cuantitativas similares al estudiado, en el presente trabajo se ha mostrado la confiabilidad de dos estimadores (BEI y $\mathrm{BEP}$ ) entre los que el método de doble muestreo con estimación por índice ha tenido un mejor ajuste con la biomasa real. Se deberían plantear estudios sucesivos complementando con otros datos con el objeto de mejorar, el uso de la altura como estimador.

\section{References:}

1. Andariese, S. y W. Covington. 1986. Biomass estimation for four common grass species in Northern Arizona Ponderosa pine. J. Range Manage. 39, pp. 472-473.

2. Aronoff, S. 2005. Remote sensing for GIS Managers. Redlands, 487. CA: ESRI Press.

3. Bianco, D.; Kraus, T.; Anderson, D. y J. Cantero. 1987. Formaciones vegetales del suroeste de la provincia de Córdoba (República Argentina) Rev. Universidad Nacional de Río Cuarto. 7(1): pp.5-66.

4. Brown, D. 1954. Methods of surveying and measuring vegetation. Commonwealth Bur. Pastures and Field Crops Bull. 42, Bradley \& Sons Ltd., Reading, England.

5. Burkart, R.; Bárbaro, N.; Sánchez, R. y D. Gómez. 2000. Ecoregiones de la Argentina. Administración de Parques Nacionales. Presidencia de la Nación. Secretaría de recursos naturales y desarrollo sustentable. PRODIA pp. 42.

6. Cabrera, A. 1976. Regiones Fitogeográficas Argentinas. En: Enciclopedia Argentina de Agricultura y Jardinería. Tomo II. Fascículo 1. ACME, Buenos Aires.

7. Cangiano, C. 1996. Métodos de Medición de Fitomasa aérea. En Ed. Cangiano, C. A. Producción Animal en pastoreo. INTA. Estación Agropecuaria Balcarce, Área producción Animal. Balcarce. Bs. As. Argentina. pp. 117-128.

8. Carrillo, J. y H. Fernández. 1988. Disponibilidad forrajera: método de doble muestreo. INTA, EERA, Balcarce. IPE 50.

9. Chiossone, G. 2011. Pastizales Naturales de Argentina. Sitio Argentino de Producción Animal. EEA. INTA. El Colorado, Formosa.

10. Díaz, R. 2007. Utilización de Pastizales Naturales Encuentro grupo editor. Editorial Brujas. Córdoba, Argentina.

11. Di Rienzo, J.; Casanovas, F., Balzarini, M.; González, L.; Tablada, M. y C. Robledo. 2010. InfoStat, versión 2010, Grupo InfoStat, FCA, Universidad Nacional de Córdoba, Argentina. 
12. Fernández, H. 2004. Estimación de la Disponibilidad de Pasto. INTA. Estación Experimental Balcarce. Área de Producción Animal. Balcarce, Buenos Aires, Argentina: 23.

13. Franco Quintero, L.; Calero Quintero, D. y C. Durán. 2006. Manejo y utilización de forrajes tropicales multipropósito. Ed. Paola A. Sanmiguel P. Palmira - Valle del Cauca - Colombia.

14. Gardner, A. 1967. Estudio sobre los Métodos Agronómicos para la Evaluación de Pasturas. Centro de Investigación y Enseñanza para la Zona Templada del instituto Interamericano de las Ciencias Agrícolas de La O.E.A. Centro de Investigaciones Alberto Boerger del Ministerio de Agricultura y Ganadería de Uruguay.- IICA- Zona SurMontevideo.

15. Guevara, J.; Gonnet, J. y O. Estévez. 2002. Biomass estimation for native perennial grasses in the plain of Mendoza, Argentina. J. Arid Envier. Vol. 50, pp. 613-619.

16. Haydock, K. y N. Shaw. 1975. Comparative yield method forestimating dry matter yield of pasture. Australian. Journal of Experimental Agriculture and Animal Husbandry. Vol.15, pp. 663670.

17. Hidalgo, L.; Cauhepé, M.; Viviani, E.; Galatoire A.y M. Meijome Colabelli. 1990. Evaluación de un método no destructivo para estimación de biomasa forrajera. Turrialba. Vol. 40, T. 3, pp. 403409.

18. Houghton R., Lawrence K., Hackler J. y S. Brown. 2001. The spatial distribution of forest biomass in the Brazilian Amazon: A comparison of estimates. Global Change Biology, 7:731-746.

19. INTA. 2011. Documento base de áreas estratégicas: forrajes y pasturas. Pergamino.

20. Jarsum, B. 1993. Plan Mapa de Suelos. Hoja 3363-20, en: Carta de Suelos de la República Argentina. Ucacha. INTA-Agricultura, Ganadería y Recursos Naturales. 72pp. Córdoba (Argentina).

21. Johnson, P.; Johnson, C. y N. West. 1988. Estimation of phytomass for ungrazed crested wheatgrass plant using allometric equations. J. Range Manage. Vol. 41, pp. 421-425.

22. León, R. y D. Anderson. 1983. El límite occidental del Pastizal pampeano. Tuexenia 3: pp.67-83.

23. Lillesand, T.; Kiefer, R. y J. Chipman. 2014. Remote sensing and image interpretation, 720. 7th ed. West Sussex: John Wiley \& Sons.

24. Luti, R; Solís, A.; Galera, M.; Ferreyra, N.; Mores, M.; Berzal, M.; Herrera, M. y J. Barera. 1979. Vegetación. Geografía física de la Provincia de Córdoba. En: Vásquez, J.B.; R. Miatello y M. Roque (eds.). Boldt. Buenos Aires (Argentina). Pp. 297-368. 
25. Medina, G.; García, R.; Gutiérrez, L.; Echavarría Chaireza, R.; Amador Ramírez, M. y A. Ruiz Corral. 2009. Estimación de la producción de forrajes con imágenes de satélite en los pastizales de Zacatecas. Técnica Pecuaria. México. Vol. 47. P 2, pp. 135-144.

26. Menghi, M.; Montani, N.; Mónaco, N.; Rosa, M. J. y M. Herrera. 1998. Diversidad y producción primaria en un pastizal inundable no pastoreado en la estepa pampeana (Argentina central). Pastos. 28 (1), 51-67.

27. Menghi, M.; Seiler, R.; Montani, N.; Mónaco, N. y MJ. Rosa. 2000. Variación anual e interanual de la producción de un pastizal inundable en la estepa pampeana (Argentina central). Relación con la precipitación y temperatura. Pastos. XXX (1): 227-240.

28. Millapán, L. 2006. Estimación de biomasa aérea en pasturas templadas de sistemas lecheros pastoriles. Trabajo final presentado para acceder al título de Especialista en Producción lechera en sistemas Argentinos. Universidad de Buenos Aires, área Producción Animal.

29. Millapán, L; Rossi, J.; Kerekes, M.; Acosta, G. y A. Ayala Torales. 2009. Estimación de fitomasa en pasturas templadas por observación visual y uso de pasturómetro. Revista Argentina de Producción animal. Vol. 29, T.1, pp. 408-409.

30. Moliterno, E.A. 1997. Nota técnica Cangué. No 10.

31. Mónaco, N., Rosa, MJ, Santa, V, Heguiabehere, A. e I. Barbero. 2014. Ajuste del método de doble muestreo para determinar biomasa en la naciente del Arroyo Chucul (Córdoba, Argentina). XXVI Reunión Argentina de Ecología. Comodoro Rivadavia Chubut

32. Mónaco, N.; Rosa, MJ.; Santa, V.; Autrán, V. y A. Heguiabehere. 2015. Utilización de estimadores para determinación de biomasa a campo. European Scientific Journal. Vol. II, N 33: 296-310. ISSN 1857-7431.

33. Pucheta, E; Ferrero, E.y L. Schneider. 2004. Modelos de regresión para la estimación de biomasa aérea en un pastizal de montaña de Pampa de Achala (Córdoba, Arg.). Agriscientia. Vol. XXI (1): 23-30.

34. Pueyo, J.M., Lacopini, L.; Fonseca, J.; Burns, J.; Bonini, Y; Ludi, R. y R. Grancell. 2005. Medición de la producción primaria del pastizal natural en el centro-norte de la provincia de Entre Ríos. Consultado 17/06/2015. En: Sitio Argentino de Producción Animal.www.producción-animal.com.ar

35. Ramírez Náder, L.; Ararat, JE; Morales, HELL y E. Quinceno. 2008. El reconocimiento de la condición de los pastizales. Estrategia de apoyo al desarrollo de sistemas sostenibles de producción ganadera. 
Univ. Nac. de Colombia sede Palmira. Fac. de Ciencias Agropecuarias, Dpto. de Ciencia Animal.

36. Redmann, R. 1992. Primary Productivity. En: Coupland. RT (eds.) Natural Grasslands Introduction and Western Hemisphere. Elsevier. New York. pp. 75-93.

37. Reese, G.A.; Bayn, R. y N. West. 1980. Evaluation of doublesampling estimators of subalpine herbage production. J. Range Manage. Vol. 33, pp. 300-306.

38. Richards, J. A. y Jia, X. 2006. Remote sensing digital image analysis: An introduction. Berlin: Springer Verlag.

39. Rodríguez, R. 1997. Estudio hidrogeoquímico como base para la planificación de uso de los recursos hídricos de la cuenca alta del arroyo Chucul, departamento Río Cuarto, Córdoba, Argentina. Tesis de licenciatura. UNRC. Córdoba (Argentina).

40. Sala, O.; Biondini, M. y M. Lauenroth. 1988. Bias in estimates of primary production: An analytical solution. Ecological Modelling Vol. 44, pp. 43-55.

41. Sala, O. y Austin, A. 2000. Methods of estimating aboveground net primary productivity. Pp. 31-43 en: Methods in Ecosystem Science. Springer. New York. Sala, O.E.; Jackson, R.B.; Mooney, H.A. \& Howarth, R.H. (Eds.).

42. Scarabotti, D.; Feldman, S. y R. Refp. 2002. Evaluación de distintos métodos para estimar la biomasa aérea en macollas de Spartina argentinensis. Parodi. PASTOS, Vol. XXXII, T 1, pp. 69-79.

43. Singh, J.; Lauenroth, W. y R. Steinhorst. 1975. Review and assessment of various techniques for estimating net aerial primary production in grassland from harvest data. Botanical Review. Vol. 41, pp. 181-232

44. $\mathrm{t}^{\prime}$ Mannetje, L. 1978. Measuring of vegetation and Animal Production of Grasslands Bull. 52 Common wealth Bureau of Pastures and Field Crops, England.

45. $\mathrm{t}^{\prime}$ Mannetje, L. 2000. Measuring biomass of grassland vegetation in Field and Laboratory Methods for Grassland and Animal Production, Edited by, L. t'Mannetje and R.M. Jones. cap. 7.

46. Turner G.; Gardner H. y V. O'Neill. 2001. Landscape Ecology in Theory and Practice, Ed. Sprinnger, New York, USA, 401 p.

47. Valencia Gutiérrez, E. 2013. Manual de Manejo de Parcelas en Pastoreo.

http://avalon.cuautitlan2.unam.mx/vaquillas/manuales/manual_pastor eo.pdf. Consultado el: 19/09/2015. 